\title{
Identificación metalográfica de fases en una junta soldada de acero estructural microaleado y su influencia en el mecanismo de fractura
}

\author{
G.O. Porras-Arévalo*, L.C. Franco-Blanco*, O.J. Gómez-Moreno*y D.E. Quinto*
}

\begin{abstract}
Resumen Este estudio pretende caracterizar las fases presentes a través de las zonas de una junta soldada de acero estructural y describir el mecanismo de fractura, ofreciendo un soporte a la industria de la construcción metálica. En la mayoría de los casos, la utilización de la soldadura en este tipo de construcción se asocia, por los diseñadores estructurales, con la fragilidad de su estructura, con la baja tenacidad y con un alto riesgo de colapso por la transición dúctil - frágil.
\end{abstract}

Palabras clave Juntas soldadas. Acero estructural. Fractura. Caracterización.

\section{Metallographic identification of phases in a welded joint of microalloyed structural steel and influence in the fracture mechanism}

\begin{abstract}
This study seeks to characterize the present phases through welded joint zones of structural steel and to describe the fracture mechanism, offering a support to the metallic construction industry. In most cases, structural designers associate weldments in this construction type with the brittleness in its structure, with the drop toughness and with a high collapse risk for the ductile-brittle transition.
\end{abstract}

Keywords Welded joint. Structural steel. Fracture. Characterization.

\section{INTRODUCCIÓN}

La estructura metálica es una alternativa de diseño y construcción que se ha venido desarrollando en Colombia, desde hace algunas décadas, mostrando una tendencia creciente como solución estructural, que hoy en día se consolida como una opción viable para su ejecución. El actual incremento en la utilización de esta opción estructural hace que, tanto ingenieros diseñadores y de soldadura, aumenten sus niveles de comunicación para garantizar así que sus sofisticados diseños, presenten excelente desempeño en operación y competitividad económica ${ }^{[1]}$. La variedad de aplicaciones industriales de los sistemas de soldadura han llegado a sustituir en el sector de la construcción, en la mayoría de los casos, al tradicional atornillado y remachado, no solamente porque significa una operación más sencilla y rápida sino que, además, el proyectista puede combinar ilimitadamente chapas, láminas, barras, tubos, perfiles, etc., para aplicar un sin número de posibilida- des de diseño que permitirán mejorar las relaciones resistencia/peso, rigidez/peso ${ }^{[2]}$, con una mayor viabilidad económica.

Con todas las ventajas y beneficios que tiene intrínsecamente la soldadura en la construcción metálica $^{[3]}$, se ha percibido que los ingenieros de diseño en la construcción civil colombiana no tienen suficiente información sobre el proceso constructivo con este tipo de elemento de unión, no aprovechando sus cualidades por falta de garantías y por la misma cultura de diseño en concreto fundamentada desde las aulas de la universidad ${ }^{[4]}$.

Se pretende dar a conocer las recientes investigaciones y consultar las inquietudes más acentuadas y con menos grado de investigación en el país, para que desde la óptica de la ingeniería metalúrgica, ayudemos a buscar el mejor aprovechamiento de las bondades ofrecidas por esta alternativa de construcción.

Investigaciones recientes buscan aclarar dudas con respecto al desempeño de este tipo de juntas,

${ }^{*}$ Universidad Industrial de Santander, Bucaramanga (Colombia) Escuela de Ingeniería Metalúrgica y Ciencia de Materiales. 
Identificación metalográfica de fases en una junta soldada de acero estructural microaleado y su influencia en el mecanismo de fractura G.O. Porras-Arévalo, L.C. Franco-Blanco, O.J. Gómez-Moreno y D.E. Quinto

frente a condiciones de corrosión en rendijas; también se ha comparado las propiedades mecánicas de una junta soldada con su análoga en juntas atornilladas y se está desarrollando la evaluación de la soldabilidad de acero estructural de bajo carbono AWS D1.1, de varillas de refuerzo aplicando el código AWS D 1.4 y de aceros utilizados bajo carga dinámica AWS D 1.5, con el único fin de fortalecer esta gran debilidad de la Construcción Colombiana.

Este trabajo presenta los resultados de la caracterización metalográfica de una junta soldada de acero estructural ASTM A 588 Grado B, usando el electrodo revestido (SMAW) como proceso de soldadura; examina la dependencia de la resistencia de la junta soldada frente a su microestructura; también, se analiza el modo de fractura de una probeta Charpy con entalla en V.

\section{MATERIALES Y PROCEDIMIENTO EXPERIMEN- TAL}

Se utilizó un acero microaleado estructural laminado en caliente ASTM A-588 ${ }^{[5]}$, cuya composición química es: 0,12 C / 0,36 Si / 1,03 Mn / 0,002 S / $0,014 \mathrm{P} / 0,029 \mathrm{~V} / 0,004 \mathrm{Nb}$, en porcentaje en peso; se seleccionó este acero por la creciente demanda de aceros con propiedades de mayor resistencia para las estructuras modernas y se usó el SMAW como proceso de soldadura, por ser el más utilizado en la industria de la construcción metálica en Colombia, con una entrada de energía promedio entre la primera y última pasada de 95,3-114,33 kJ/cm, respectivamente. El valor estimado de carbono equivalente es de 0,2923\%.

En este estudio, se pretende caracterizar las fases presentes en una junta soldada de acero estructural y observar la influencia que tiene en el mecanismo de fractura. Para tal fin se aplicó un registro de la calificación de un procedimiento (PQR) precalificado para evaluar el desempeño de un soldador WPQ, bajo los criterios de aceptación del código de soldadura para acero estructural AWS D1.1. ${ }^{[6]}$

La caracterización metalográfica de las fases presentes se realizó en el microscopio óptico (MO) ${ }^{[7]}$, atacando ${ }^{[8]}$ las muestras con nital al $4 \%$; la junta soldada se puede dividir en tres zonas metal base, metal de soldadura y la zona afectada térmicamente (HAZ).

Las mediciones de los valores de microdureza, se tomaron en la zona transversal de las probetas, bajo una carga de $100 \mathrm{~g}$ en tres barridos distribuídos de la siguiente manera: superior, medio e infe- rior (Fig. 1). Para el ensayo Charpy se referenció la norma ASTM E 345-85, la cual se usó para estimar la tenacidad, a $25^{\circ} \mathrm{C}$, de la junta soldada. El ensayo de tensión y doblez se llevó a cabo bajo las especificaciones del AWS D 1.1 -2002 para medir la ductilidad y sanidad de la junta soldada. Posteriormente a realizar estos ensayos se caracterizó la superficie de fractura mediante el uso del microscopio de barrido electrónico.

\section{RESULTADOS Y DISCUSIÓN}

\subsection{Microdureza}

La dureza aumenta significativamente a través de la HAZ y de la línea de fusión al lado del metal de la soldadura, con un máximo de $298 \mathrm{HV}$, aproximadamente a $200 \mu \mathrm{m}$ de la línea de fusión. Esta región corresponde a la del material que había experimentado la recristalización durante el proceso de la soldadura. Se elimina la posibilidad de existencia de fases frágiles como bainita o martensita, las cuales oscilan entre 500-900 HV y son indeseables en las aplicaciones de la soldadura como elemento de unión en estructuras sismorresistentes.

\subsection{Microestructura y macroataque}

La microestructura de este acero microaleado, conformada de ferrita y perlita como se muestra en la figura 2A). Esta muestra longitudinal permite observar las líneas de deformación, con un grano de tamaño número 7 , siendo su dureza promedio de $175 \mathrm{HV}$.

Línea de recocido: es la primera zona en enfriarse, lo que hace que exista un afino de grano con la presencia de una nueva ferrita equiaxial y

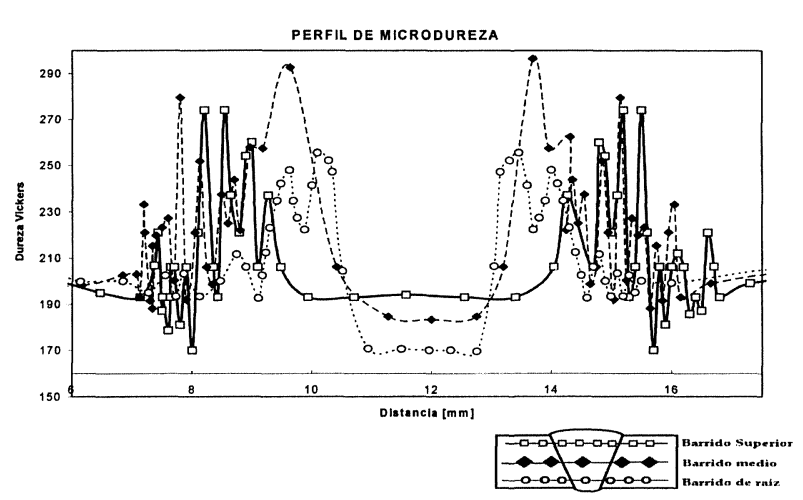

Figura 1. Microdureza junta de Acero ASTM A-588 Gr. B.

Figure 1. Microhardness in a welding joint of Steel ASTM A$588 \mathrm{Gr}$. B.

Rev. Metal. Madrid Vol. Extr. (2005) 137-142 
perlita fina. (Figs. 2B) y 2G)). Por esta razón, la dureza sufre un incremento.

Línea de fusión: en esta zona tiene lugar la transformación eutectoide, el producto es una perlita fina, debido a que el tiempo de sostenimiento a esta temperatura es corto, lo que hace sea limitado el crecimiento de grano (Figs. 2C) y 2F)).

La HAZ presenta una microestructura compuesta por: ferrita proeutectoide (fase clara) en formas alargadas y de mayor tamaño, además de una mayor cantidad de perlita fina (fase oscura) en comparación con la zona de recocido, originando un incremento en dureza; a su vez, se observó que la ferrita de la zona de recocido cambia su forma equiaxial a acicular y en placas.

El metal de soldadura (Fig. 2J)) presenta su microestructura compuesta por ferrita proeutectoide en forma de granos columnares, que siguen la dirección de enfriamiento. Además, se presenta la estructura Widmanstätten (Figs. 2I) y 2E)), en la que, a su vez, la cementita está presente en forma de láminas, que sugieren una estructura perlítica laminar y presentan una morfología y dirección diferente a la de las demás zonas descritas anteriormente. Estos resultados están a acorde con la composición química de las diferentes pasadas (Tabla I).

La estructura de la HAZ en el metal de soldadura, con cordones de subsecuentes, presenta una microestuctura típica de este acero en estado normalizado, con un afinamiento en el grano. Los granos aparecen, primero, en la línea de fusión, en donde la temperatura es relativamente baja, y crecen con rapidez diferente.

\subsection{Ensayo de doblez y tensión}

Su aprobación se hizo, bajo el criterio de aceptación sección 1.9.8.3.3 del AWS D 1.1. Además, se atacó la probeta de doblez y se llevó al microscopio de barrido electrónico (Figs. 3a) y 3b)), donde se
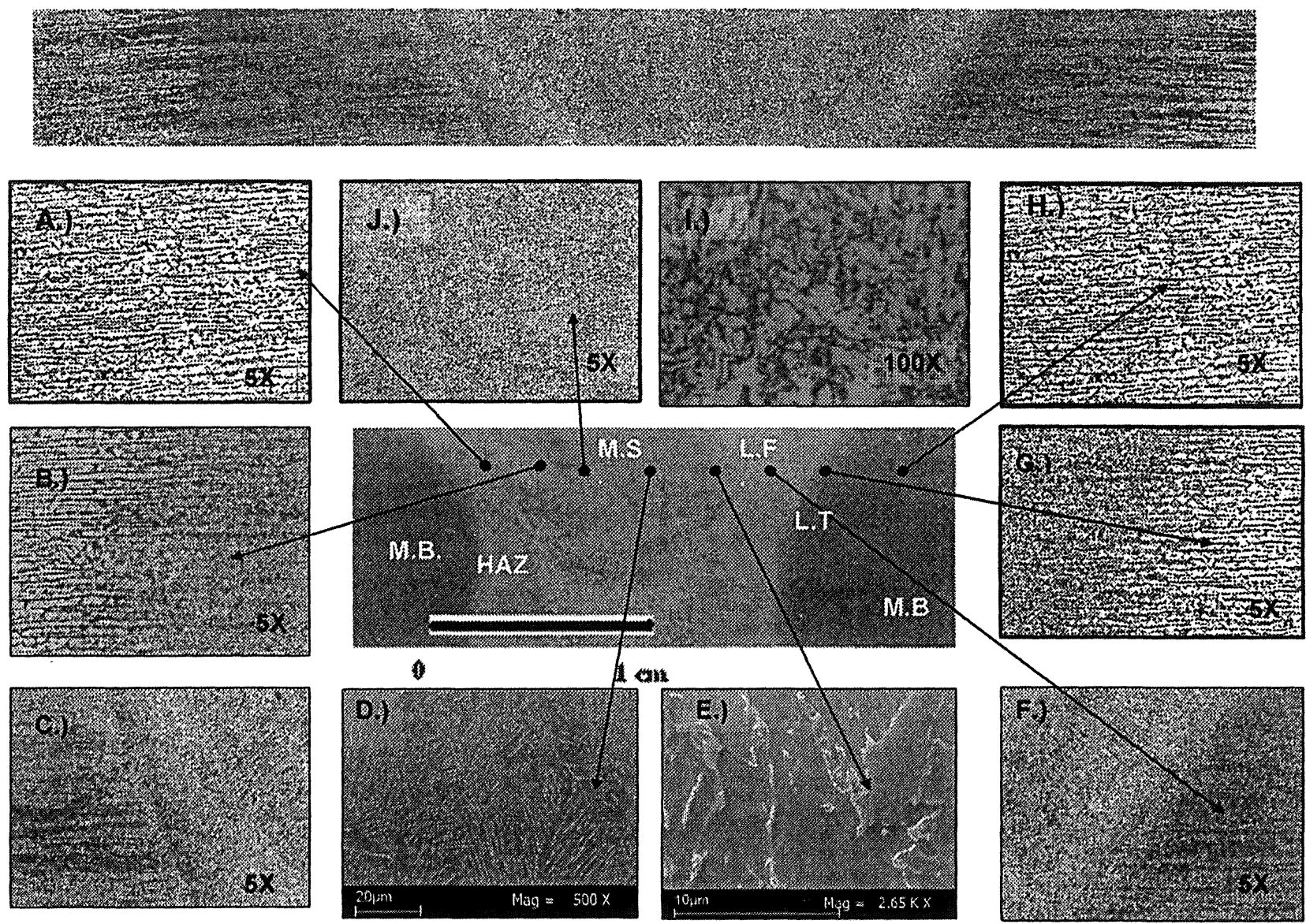

Figura 2. Macroataque de la junta soldada y micrografía de las diferentes zonas presentes en la junta. A) y H) Metal base. B) y G) Línea de transición metal base - zona afectada por el calor (HAZ). C) Zona afectada por el calor. (HAZ). D) y E) Metal de soldadura a 500X y $2650 \mathrm{X}$ correspondientemente.

Figure 2. Macroetching of the welded joint and micrographic of the different present areas in the joint. A) and H) Base metal. B) and G) Transition line base metal - heat affected zone (HAZ), E) and C) Heat-affected zone (HAZ). D) and I) Welding metal at 500X and 2650X correspondingly. 
Identificación metalográfica de fases en una junta soldada de acero estructural microaleado y su influencia en el mecanismo de fractura G.O. Porras-ArÉVAlo, L.C. Franco-Blanco, O.J. Gómez-Moreno y D.E. Quinto

Tabla I. Composición química del los pases de soldadura, \% en masa

Table I. Chemical composition of the welding passes, mass \%

Composición química del cordón de soldadura

SMAW : $V=22,5$ Volt; $A=110$ Amp

\begin{tabular}{lcccccccccccc}
\hline Pase & $\mathbf{C}$ & $\mathrm{Mn}$ & $\mathrm{Si}$ & $\mathrm{S}$ & $\mathbf{P}$ & $\mathbf{V}$ & $\mathrm{Nb}$ & $\mathrm{Cr}$ & $\mathrm{Ni}$ & $\mathrm{Cu}$ & $\mathrm{Sn}$ & $\mathrm{Mo}$ \\
\hline Penetración & 0,084 & 1,286 & 0,412 & 0,0284 & 0,0308 & 0,0058 & 0,0008 & 0,11 & 0,08 & 0,062 & 0,003 & 0,008 \\
Relleno & 0,12 & 0,622 & 0,521 & 0,022 & 0,013 & 0,00435 & 0,0006 & 0,0825 & 0,06 & 0,429 & 0,00225 & 0,006 \\
Presentación & 0,12 & 0,574 & 0,54 & 0,0268 & 0,013 & 0,00145 & 0,0002 & 0,0275 & 0,02 & 0,443 & 0,0075 & 0,002 \\
\hline
\end{tabular}
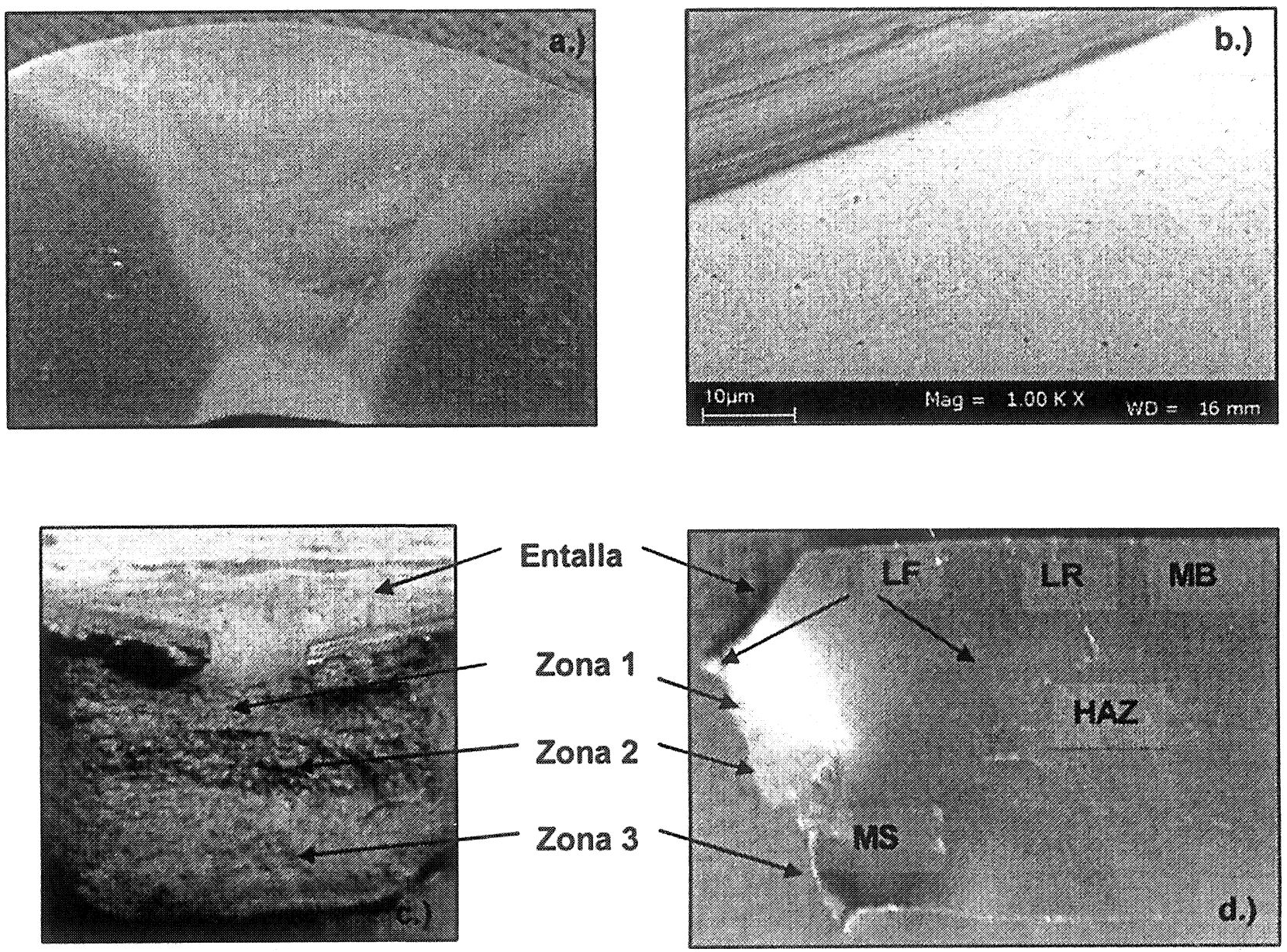

Figura 3. a.) Macroataque probeta ensayo doblez. b.)Superficie bajo SEM probeta doblez. c.) Superficie de fractura probeta ensayo Charpy. d.) Trayectoria de la propagación de la grieta.

Figure 3. a.) Macroetching of the side-bend test specimen, b.) Surface on SEM of the side-bend test specimen. c.) Surface of fracture of the Charpy side-bend test specimen. d.) Propagation path of the crack.

aprecia que la superficie transversal está libre de grietas, evidenciando que la ductilidad del material se conserva en la zona de la soldadura.

El esfuerzo de tensión que se obtuvo es mayor que el valor especificado para el metal base, lo que indica que no falló por la soldadura.

\subsection{Fractografía}

De acuerdo a la superficie de fractura presentada en una probeta de una junta soldada con entalla en $\mathrm{V}$, ubicada en la línea de fusión (Fig. 5), se observan tres zonas identificables en la figura 1, 

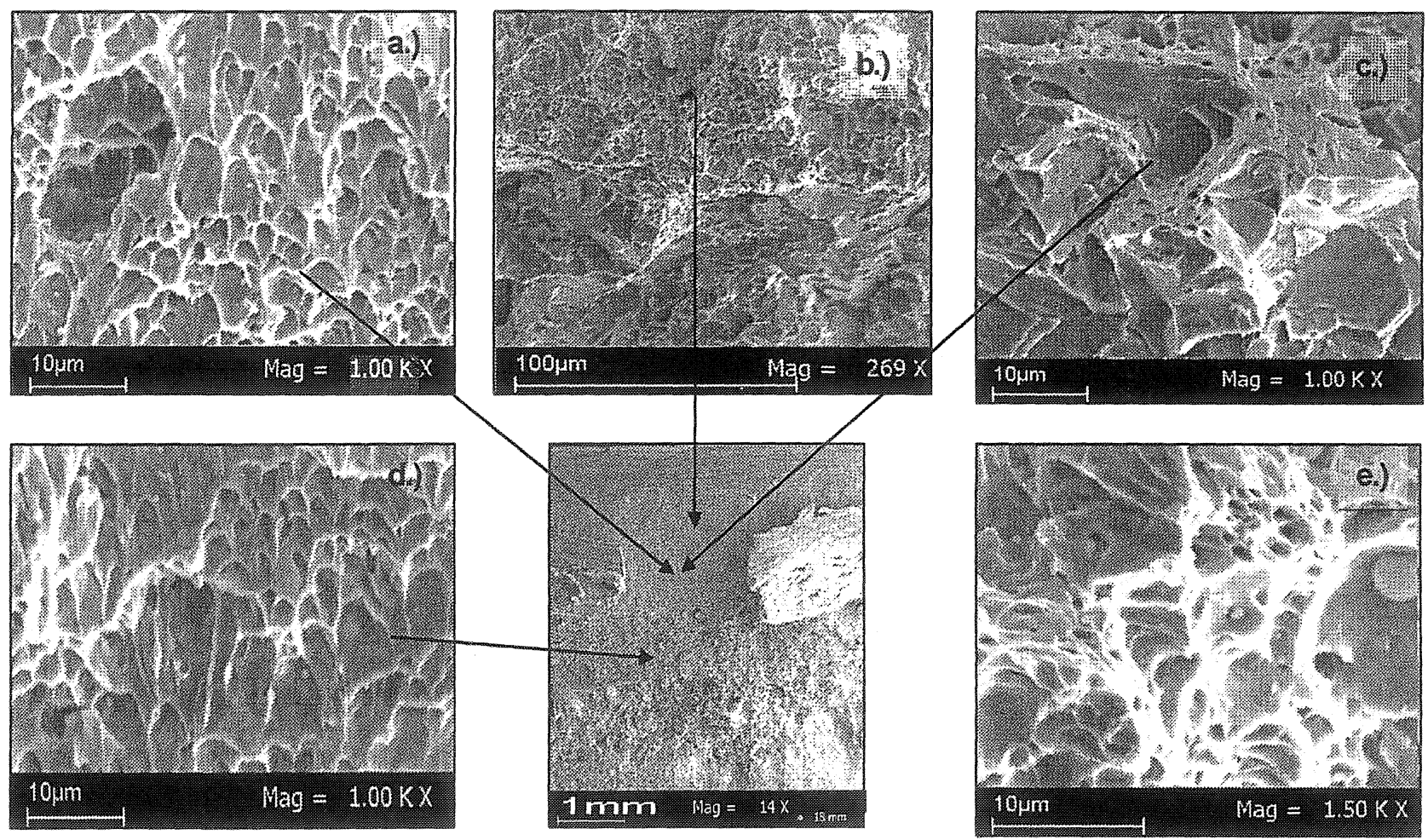

Figura 4. Fractografía en el SEM a.) Zona 1: fractura dúctil, b.) Zona de transición, c.) Zona 2: fractura en clivaje d.) Zona 3 inicial, y e.) Zona 3 final: dúctil.

Figure 4. Fractographys in the SEM, a.) Zone 1: ductile fracture MVC, b.) Transition zone, c.) Zona 2: fracture in cleavage, d.) Zone 3 initial, and e.) Zone 3 final: ductile.

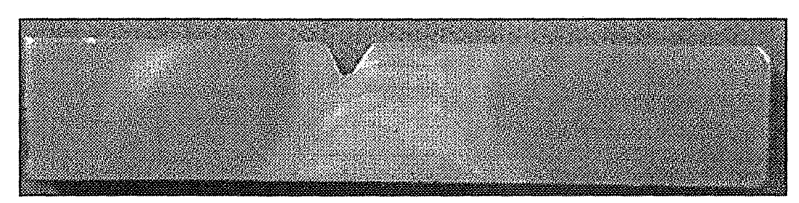

Figura 5. Ubicación de la entalla en la probeta Charpy.

Figure 5. Location of the notch in Charpy test.

donde se muestra el avance de la grieta en la junta soldada, del siguiente modo:

\section{Zona 1:}

Esta zona es continua a la entalla y su fractografía consiste en:

fractura dúctil: se aprecia la formación de la coalescencia de los micro hoyuelos MVC, por sus iniciales en inglés micro void coalescence. Esta fractografía es característica de un metal dúctil y, en esta zona, los hoyuelos muestran un mayor alargamiento, debido a su alto flujo plástico; se observa que su mayor deformación se presenta en la parte inicial del avance de la grieta ${ }^{[9]}$.

Esta morfología se atribuye a la capacidad que tiene el material, en un principio, de absorber energía.

\section{Zona 2:}

Presenta fractura en clivaje, estando unidas algunas facetas de clivaje por MVC, detectándose una deformación plástica; a este modo de fractura inestable se le denomina "seudoclivaje"; atribuible éste, al gradiente microestructural y a la velocidad de deformación, que hacen que el material no esté en capacidad de absorber más energía. Finalmente, se observó que la grieta tiende a propagarse por la zona de menores propiedades de absorción de energía.

\section{Zona 3 inicial:}

Está compuesta por MVC de hoyuelos alargados, los cuales nos indican el esfuerzo y la dirección de avance del péndulo en la probeta; éstos, presentan morfología ovalada corroborándose al comparar la fractografía de la zona 1 y la zona 3 en la figura $3 \mathrm{a}$ y $3 \mathrm{c}$.

\section{Zona 3 final:}

Según clasificación de la coalescencia de los hoyuelos de Beachem ${ }^{[10]}$, la forma de éstos, en la zona cuatro, es alargada y más corta que la de los anteriores, debido a la relación de deformación. La 
forma de los hoyuelos no difiere mucho con la zona anterior, lo cual se atribuye a la deformación plástica que sufre la probeta, por el impacto proporcionado por el martillo de la máquina de ensayo Charpy.

\section{CONCLUSIONES}

El presente trabajo de investigación nos permite concluir que:

- El acero estructural microaleado presenta una tenacidad y unas propiedades mecánicas aceptables para los requerimientos en servicio de las construcciones metálicas sometidas a cargas dinámicas y estáticas.

- El análisis de la fractura permitió verificar que un $80 \%$ se produjo de manera dúctil y en el 20 $\%$ restante se presentó un seudoclivaje; atribuyen este comportamiento, a la velocidad de la propagación de la grieta.

- La HAZ consiste en una región con una considerable variedad en su microestructura y en las propiedades mecánicas. Así, la tenacidad de las diferentes zonas no puede ser representada por un único valor.

- La dirección de la propagación de la grieta depende de la diferencia de la absorción de energía de las zonas adyacentes a la línea de fusión; en particular, se observó que sigue la zona de menor absorción, en este caso el metal de soldadura.

\section{RECOMENDACIONES}

La principal recomendación, va orientada a complementar este trabajo y otros del mismo género ${ }^{[11]}$, aplicando técnicas de simulación de los tratamientos térmicos inherentes al proceso de soldadura y aplicando estándares para obtener valores de la resistencia a la tenacidad, esperando obtener resultados que permitan apreciar el efecto de la temperatura, estado triaxial de esfuerzos, discontinuidades y la velocidad de propagación de la grieta en zonas con micro estructuras homogéneas caracterizadas en el presente trabajo.

\section{Agradecimientos}

Los autores reconocen el apoyo ofrecido por la Universidad Industrial de Santander, el laboratorio de microscopia electrónica del Instituto Colombiano del Petróleo, a Servicio Automotriz Chimitá por el apoyo financiero y a la asistencia técnica en la aplicación de soldaduras y Acería de Caldas ACASA por la provisión de aceros. Finalmente, un agradecimiento especial al señor José Porras a los Ingenieros Wilson Vesga, Jorge Báez, Fabio Vargas, Martín Lizcano, Darwin Martínez y Rubiel Ordóñez.

\section{REFERENCIAS}

[1] Asociación Colombiana de Soldadura, SeminarioTaller: Actualización Tecnológica de Inspectores de Construcciones Soldadas, Bogotá, Colombia, 2000.

[2] J.M. Benhayon, III Congreso Colombiano de Soldadura y III Exposición Industrial: Colombia, 1994, Centro Argentino de Tecnología de la Soldadura (CATS).

[3] MCCORMAC, Diseño de estructuras de acero, método LRFD, Ed. Alfaomega, México, 1996, Cap. 3 y 14.

[4] Asociación Colombiana de Ingeniería Sísmica, Normas Colombianas de Diseño y Construcción Sismo Resistente, Bogotá, Colombia, Ley 400 de 1997, pp. 7-23 y sección F: Estructuras Metálicas, Capítulos F6.5 y F6.5.3.

[5] ASTM A 588/A 588 M - 88 A, Especificación estándar para aceros estructurales de alta resistencia y baja aleación.

[6] CÓDIGO AWS D1.1/2002, Código de soldadura para acero estructural, Sección 4, parte C: Performance Qualification.

[7] L.E. Samuels, Light Microscopy of Carbon Steels, ASM International, 1st. Ed. EE.UU., 1999, pp. 309 -359.

[8] G. Petzow, Metallographic Etching, ASM International, 2nd Ed., 1999.

[9] T.L. Anderson, Fracture Mechanics Fundamentals and Application, 2nd Ed., CRC Press LLC, EE.UU., 1995, pp 265-289.

[10] AMS Metal Handbook, Volume 12 Fractography, ASM Publication, United States of America, 1987

[11] R.C. Silverio, T. Moura y P. Guedes, Rev. Metal. Madrid 39 (2003) 83-89. 